\title{
West Syndrome: Clinical Characteristics, Therapeutics, Outcomes and Prognosis
}

\author{
Ernesto Portuondo Barbarrosa ${ }^{1 \star}$, Iraida de la Caridad Pérez Ferrer ${ }^{1}$, Marcos Roberto Tovani-Palone ${ }^{2^{\star \star}}$
}

${ }^{1}$ Centro Habana Teaching Pediatric Hospital, Havana, CUBA

${ }^{2}$ Ribeirão Preto Medical School, University of São Paulo, Ribeirão Preto, BRAZIL

*Corresponding Author: ernestopb@infomed.sld.cu

${ }^{\star \star}$ Corresponding Author: marcos_palone@hotmail.com

Citation: Barbarrosa EP, Ferrer ICP, Tovani-Palone MR. West Syndrome: Clinical Characteristics, Therapeutics, Outcomes and Prognosis. Electron J Gen Med. 2020;17(2):em190. https://doi.org/10.29333/ejgm/7800

ARTICLE INFO

Received: 30 Oct. 2018

Accepted: 2 Feb. 2020

\begin{abstract}
Introduction: West syndrome (WS) is the most severe, devastating and/or catastrophic epileptic encephalopathy during the lactation period. However, until now, there are few comprehensive clinical studies in this regard in Cuba.

Objective: To identify clinical characteristics of patients with WS, the related etiology, therapeutics and prognostic factors.

Methods: An observational, descriptive and retrospective study of 39 patients with WS from the Centro Habana Teaching Pediatric Hospital, Havana, Cuba, comprising the period between January 2005 and December 2016 was carried out. Clinical data were recorded for each patient by review of clinical history. Statistical analysis was performed with use of the Statistical Package for Social Sciences.

Results: The genetic, structural/metabolic etiology was predominant. Hypoxic ischemic encephalopathy (25.6\%) and neurocutaneous syndromes (17.9\%) were the cause more frequent of WS. The combined treatment of adrenocorticotropic hormone (ACTH) and vigabatrin (VGB) was used in 25 patients (64.1\%). In 18 of them (72\%) there was control of infantile spasms or reduction $\geq 50 \%$, while in 14 patients $(56 \%)$ the hypsarrhythmia disappeared in the first 6 months $(p<0.05)$. All patients that used ACTH had transient hypertension as an adverse effect of this drug. $71.8 \%$ of the patients had moderate to severe delay in psychomotor development, $35.9 \%$ were diagnosed with Lennox-Gastaut syndrome and $43.6 \%$ with other epilepsies. There was an unfavorable evolution in $74.3 \%$ of the patients. The most significant prognostic factors for unfavorable evolution were male gender symptomatic etiology, psychomotor delay and/or abnormality in the neurological examination, epileptic seizures, previous paroxysmal electroencephalogram, poor response to the treatment, persistence of hypsarrhythmia, and combination of more than three factors in $68.9 \%$ of the patients with unfavorable evolution $(p<0.05)$.
\end{abstract}

Conclusions: Our findings showed that the combined treatment with ACTH and VGB may decrease the time to disappearance of infantile spasms and hypsarrhythmia in patients with WS. Moreover, the unfavorable evolution of these patients is related with the etiology and combination of prognostic factors.

Keywords: syndrome, spasms, infantile, epileptic encephalopathy, etiology, prognosis

\section{INTRODUCTION}

West syndrome (WS) is the most severe, devastating and/or catastrophic epileptic encephalopathy during the lactation period. WS was first described by Dr. William James West in 1841 in his 4-month-old son. He reported the first evidence on infantile spasms (IS) associated with such syndrome (1-5). According to the 2010 International League Against Epilepsy (ILAE) classification (6), WS comprises a triad of epileptic spasms (ES), with hypsarrhythmia detected by electroencephalogram (EEG), and delay or regression in psychomotor development (PD), but this last characteristic is not essential for its definition.

WS, moreover, corresponds to an age-dependent epilepsy. The age of onset of this syndrome varies from 4 to 10 months, with a higher occurrence between 5 to 7 months, which can be extended until 2 years $(3,7,8)$. An incidence of 1 case of WS per 4,000 children has been estimated in the literature $(3,4,9,10)$. Such disease represents around 2 to $10 \%$ of the epilepsies in childhood and it is the most frequent encephalopathy which occurs before the first year of life (with the exception of the Ohtahara syndrome). A higher prevalence of WS has been observed in males $(3,7,8)$.

In accordance with the ILAE classification in force until 1989 (10), ES were named as infantile spasms, due to their broad association and exclusivity to WS, and occurrence in early childhood and lactation period. More recently, the ILAE classification 2010 (6) named these alterations as ES, because their relationship with other epileptic syndromes with occurrence outside of the mentioned period. Despite of this, 
the term IS (3) continues to be used when referring to spasms that occur before 2 years of age and related to the WS.

Several authors have demonstrated the existence of patients with ES without hypsarrhythmia in the interictal EEG $(11,12)$. This has been interpreted as a variant of the WS and not another type of epileptic syndrome. Consequently, in this context it is relevant to perform critical EEG to focus the differential diagnosis with other epilepsies (that occurs in similar periods) and paroxysmal non-epileptic events (11).

IS usually occur in clusters (spasms with different intensity, frequency and/or duration). They correspond, in turn, to axial contractions in flexion, extension or mixed extension-flexion, lasting from 2 to 3 seconds, which are most commonly observed during the transition from slow sleep to REM sleep. IS are subtle as the deviation of the eyes upwards, slight movements of head or elevation of the shoulders, and autonomic signs. Furthermore, they are generally symmetrical, but can also be asymmetric or focal, alternating, and associated with focal seizures. In this last case, together with a possible focal lesion or agenesis of the corpus callosum (3-5, 9,11,14-16).

Etiologically, according to the previous ILAE classification $(3,10)$ in relation to aggressions or prenatal, perinatal and postnatal antecedents, IS are classified as symptomatic, cryptogenic or probably symptomatic, (being these the most frequent) and idiopathic cases (rare).

The new ILAE classification $(6,10,14)$ proposes the use of the term genetic, structural/metabolic and unknown cause. Approximately $64 \%$ of patients with WS have known etiology (13). Currently, with the advent of neuroimaging studies with great specificity and sensitivity, such as nuclear magnetic resonance (NMR) and single positron emission tomography, and the increase in the number (around $80 \%$ ) of studies of metabolic screening and molecular genetic techniques, it has been possible to diagnose a greater number of patients with related structural/metabolic and genetic alterations (16-18). In view of this, the findings in anamnesis and the neurological and/or general physical examination (PE) abnormalities can be associated with the etiological diagnosis and the results of the complementary examinations. In this respect, the knowledge about the etiology is of great importance, since this may lead to the optimal treatment, with positive implications on the evolution and prognosis (7,16-18).

Most of the cases of WS have an unfavorable evolution, related to different prognostic factors that alone or combined influence the severity of this syndrome $(3-5,7,16)$. There are evidences about the efficacy of several drugs for the treatment of WS. However, the selection of the first choice drug is still a controversial subject. Currently, the use of vigabatrin (VGB) in combination with adrenocorticotropic hormone (ACTH) has been recommended as the most effective therapy for these patients. Other drugs, such as prednisolone (1), valproic acid (VA), benzodiazepines, vitamin B6 (VB6), topiramate (TPM), levetiracetam (LVT), and zonisamide have also shown efficacy. In addition, the ketogenic diet, intravenous gammaglobulin therapy and, surgical treatment in refractory cases and specific situations are also useful options for the treatment of such disorder, which have been used increasingly in specialized centers for epilepsy $(4,5,7,8,14,16,19-31)$.

Up till now, there are few comprehensive clinical studies about this subject matter from Cuba. Thus, the present research intents to identify the clinical characteristics of patients with WS, the aspects inherent to the related etiology, semiology, EEG results, the used medications and their adverse reactions, as well as to determine the prognostic factors that can influence the evolution of these patients.

\section{METHODS}

\section{Design, Context and Participants}

An observational, descriptive and retrospective study of patients with WS from the Centro Habana Teaching Pediatric Hospital, Havana, Cuba, comprising the period between January 2005 and December 2016 was carried out. Informed consent of parents and/or persons responsible, and approbation of the ethics committee of the institution were obtained. Patients were treated on an outpatient basis or by hospital admission according to their individual needs.

All patients with IS and hypsarrhythmia on EEG beginning before two years of age, with or without alteration in PD, diagnosed with WS, admitted, treated and followed up in Neuropediatric consultation by more than two years were included in this study. We excluded patients who left the follow-up and /or had clinical history with insufficient data. Of the total of 45 patients, 39 ( 22 boys and 17 girls) were included. The other six were excluded.

\section{Variables and Interventions}

The following data were recorded for each patient: age of onset of IS, sex, semiological characteristics of IS (frequency, type, symmetric/asymmetric), description of EEG alterations, abnormalities in the neurological examination during PE, PD prior to initiation of IS or a posteriori, related etiology, clinical evolution towards other types of epileptic seizures and/or epileptic syndromes recognized by ILAE, related comorbidities (intellectual disability and autistic spectrum disorder (ASD)), the used therapeutic options, response to the treatment and adverse reactions, prognostic factors and unfavorable evolution.

The age of onset of IS was subdivided into three categories, i.e., before 4 months ( $<4$ months), between 4 and 8 months, and after 8 months (> 8 months). According to the ILAE (6) classification, this subdivision should be used when referring to genetic and structural/metabolic etiology, and unknown cause. In this study, all patients with relevant prenatal, perinatal and postnatal antecedents, presence of clinical manifestations and data on evaluation of PE were subjected to biochemical studies, which included glutamic-oxaloacetic transaminase, glutamic-pyruvic transaminase, lactic acid, lactic acid dehydrogenase and ammonia. In addition, the neurometabolic study was extended to the qualitative study of amino acids in urine and quantitative in blood, including serology for Cytomegalovirus and indirect immunofluorescence for detection of Toxoplasma gondii. A neuroimaging study was performed in specific cases with use of transfontanellar ultrasound, computerized axial tomography and magnetic resonance imaging of the skull. When a cause was not determined, it came to be called unknown (3-5,7-9,14,16-18).

The definition of related concepts was based on the previous experience of different authors $(3-5,7,8,11,14,16,32$, 33): 
- EEG hypsarrhythmic interictal: characterized by the presence of paroxysmal slow wave discharges, spikes and polyspikes with a great amplitude of duration and variable location (symmetrical, asymmetric or alternating locations), with or without paroxysmal voltage attenuation, which give it a pseudoperiodic aspect. There is also a highly disorganized base rhythm.

- Subclinical spasms: comprise the occurrence of generalized slow waves of high amplitude, fast and rhythmic activity called spindle - like, due to its resemblance to sleep spindles with diffuse voltage attenuation.

- Prognostic factors: male gender, presence of relevant prenatal, perinatal and/or postnatal antecedents prior to initiation of IS including paroxysmal EEG, delayed PD and/or abnormality in the neurological PE, neonatal epileptic seizures or before onset of IS, and onset of IS before 4 months; time lost prior to treatment initiation or diagnosis greater than 1 month, known etiology, no response to treatment with monotherapy (one drug) or bitherapy (2 drugs), hypsarritmic EEG persisting after 6 months of initiation of therapy and combination of more than three prognostic factors.

- Unfavorable evolution: persistence of spasms and hypsarrhythmia on EEG after 6 months of treatment, little response to bitherapy, regression of $\mathrm{PD}$ or, moderate or severe PD delay and/or abnormality in the neurological PE, moderate or severe intellectual disability (ID) and/or ASD, evolution to Lennox-Gastaut syndrome (LGS) or other epilepsies and death.

- Adverse reaction to medication: irritability, weight gain, immunosuppression or added infections, transient arterial hypertension (TAH), hydroelectrolytic disorders and hyperammonemia.

All patients underwent EEG with sleep deprivation, before and after the start of treatment (15 days, 1 month, every 3 months during the 1st year). The aim of this step was to evaluate the response of the patients to the treatment according to their clinical evolution. It was observed if the hypsarrhythmia disappeared before 2 months ( $<2$ months), between 3 and 5 months or persisted after 6 months (> 6 months). According to the clinical characteristics, they were evaluated by the ophthalmology, genetics and child psychiatry services. 36):

Scheme of treatment used according to etiology (19-31,33-

- ACTH in combination with VGB was used in cases of known or symptomatic/structural etiology, except to neurometabolic disease with absence of symptoms and/or acute infectious signs, dermatological conditions, TAH, liver or kidney damage. ACTH was given intramuscularly at a dose of $0.0125 \mathrm{mg} / \mathrm{kg} /$ day intramuscular (with presentation of $1 \mathrm{mg} / 1 \mathrm{ml}$ ampules), at intervals of alternate days in the first and second week. According to the response its use was of two times during the third week and once in the other weeks without exceeding six weeks (the most frequent dose used was 2 tenths). VGB was used at doses of 50 to $200 \mathrm{mg} / \mathrm{kg} /$ day every 12 hours, with an initial dose of $25 \mathrm{mg} / \mathrm{kg} /$ day increasing every 3 days (maximum dose $3000 \mathrm{mg}$ - 6 tablets). If a good response was observed between 4 and 6 weeks, the treatment was continued for 4 to 9 months. In this case, VGB was replaced (taking into account clinical characteristics and EEG) for Valproate sodium (VS) at a dose of 20 to $60 \mathrm{mg} / \mathrm{kg} /$ day in 2 or 3 subdoses, increasing at a rate of $5 \mathrm{mg} / \mathrm{kg} /$ day every 5 days. If there was any contraindication to the use of $\mathrm{ACTH}$, the treatment was started only with use of VGB.

- If there was no response to VGB in the first 4 to 6 weeks (no disappearance of IS and persistence of hypsarrhythmia on EEG, or IS were not reduced in more than $50 \%$ of the initial number), we used additionally VS, or VGB was replaced by VS with consent of parents and/or guardians. In case of no response, TPM was used at doses of 3 to 25 $\mathrm{mg} / \mathrm{kg} /$ day in 2 sub-doses, increasing at a rate of 0.5 to 1 $\mathrm{mg} / \mathrm{kg} /$ day every 10 days with a maximum dose of $400 \mathrm{mg}$. In addition, LVT was also used at doses 30 to $60 \mathrm{mg} / \mathrm{kg} / \mathrm{day}$ in 2 sub-doses, increasing at a rate of $10 \mathrm{mg} / \mathrm{kg} /$ day every 7 days with a maximum dose of $3000 \mathrm{mg}$. In case of no response, clobazam (CLB) at doses of 0.1 to $1 \mathrm{mg} / \mathrm{kg} /$ day in 2 to 3 sub-doses or clonazepam (CLN) at a dose of 0.025 $\mathrm{mg}$ to $0.2 \mathrm{mg} / \mathrm{kg} / \mathrm{day}$ in 2 or 3 sub-doses were used.

- In case of unknown etiology/probably symptomatic, Down syndrome or suspect of metabolic error, treatment with VB6 was instituted at a dose of $300 \mathrm{mg}$ per day in 3 subdoses. The response to medications was evaluated during the first 4 days. If there was no alteration in IS or their increase, the same therapeutic scheme of known or symptomatic/structural cause was used. In case of suspect or confirmation of inborn error of metabolism, the treatment with VGB was used. Depending of the achieved response, other drugs were used with exception of VS, which can be associated with complications, such as metabolic disease of urea cycle disorder, mitochondrial disease or hyperglycinemia without ketosis.

We did not use other corticosteroids or other therapeutic options, the drugs were used in accordance with the pharmaceutical availability and the treatment was adapted according to evolution to other epileptic syndromes. The use of ACTH was performed under medical supervision and hospital admission. The response to the treatment was evaluated based on the following parameters: disappearance of IS and hypsarrhythmia, free of IS but with hypsarrhythmia, reduction of more than $50 \%$ of IS and persistence of hypsarrhythmia, reduction of IS less than $50 \%$ and persistent hypsarrhythmia (without response to the treatment).

\section{Statistical Processing}

Data were collected and, tables and graphs were made in Microsoft Excel ${ }^{\circledR} 2010$ (Microsoft Corporation, Redmond, WA, USA). Variables were expressed in absolute frequency. Some quantitative variables were also expressed in percentages. Chi2 test was used for statistical analysis. $\mathrm{P}<0.05$ was considered significant and confidence intervals at $95 \%$ were calculated. The statistical analysis was performed with use of the Statistical Package for Social Sciences (SPSS), version 16.0 program for Windows ${ }^{\circledR}$.

\section{RESULTS}

Among the 39 studied patients, a higher number of males (56.4\%, 22 males and 17 females) was observed. Regarding the age of onset of IS, a higher frequency was observed in the age range between 4 and 8 months ( $n=23,58.9 \%)$, while before 4 


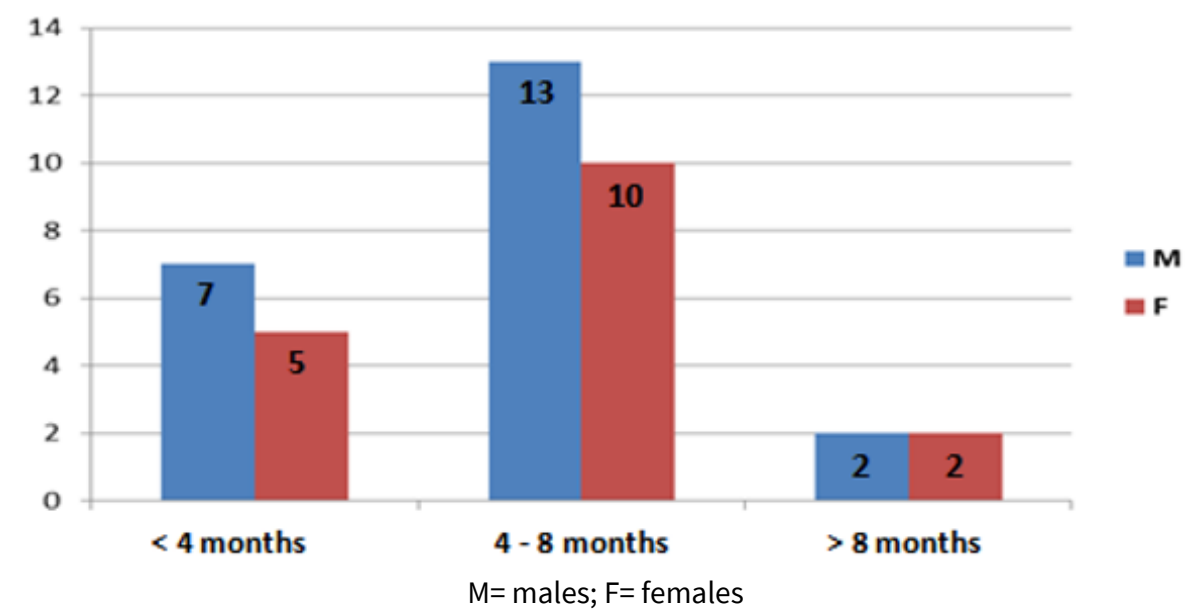

Figure 1. Distribution of patients according to age of onset of IS and sex. Hospital Pediátrico Docente Centro Habana (Centro Habana Teaching Pediatric Hospital) from 2005 to 2016

Source: Clinical history

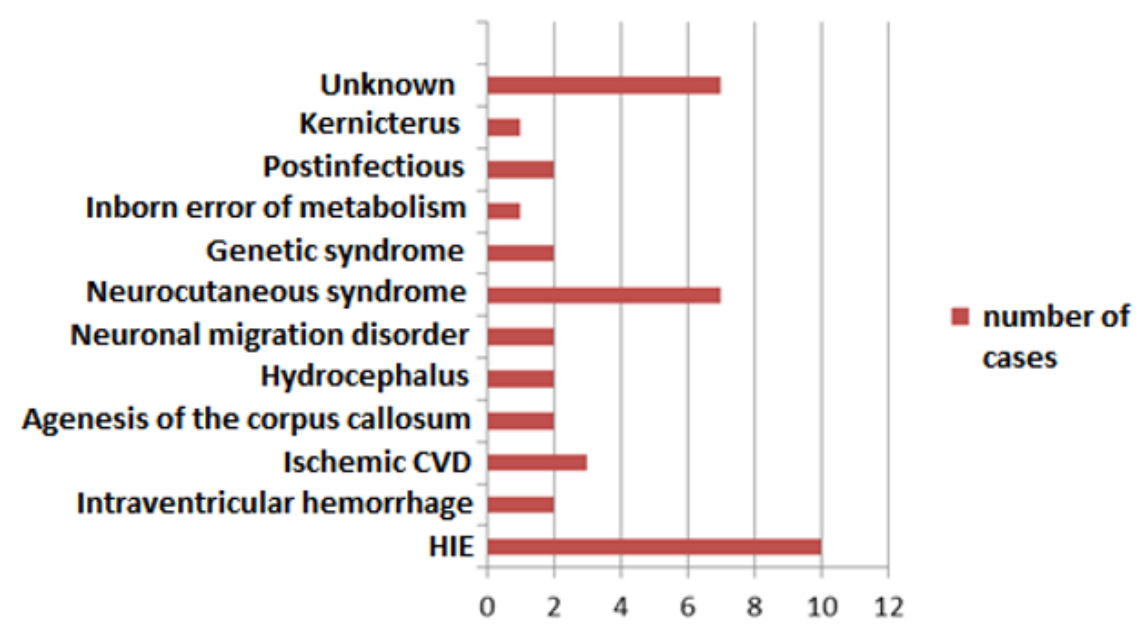

$C V D=$ Cerebrovascular disease; $\mathrm{HIE}=$ Hypoxic ischemic encephalopathy

Two patients had (bacterial meningoencephalitis + hydrocephalus), (HIE+ intraventricular haemorrhage)

Figure 2. Etiologies related to West syndrome

Source: Clinical history

months of age we had 12 cases (30.7\%) registered. There was no statistical association between these variables (Figure 1).

There was a predominance of structural/metabolic etiology. This etiology was observed in registered cases (30.7\%). A genetic cause due to the INV 21 (P112-q21) 13 pst 5tk and Down syndrome or trisomy 21 were verified in two patients, and no known cause was defined to seven. Among al patients with WS in the study, $32(82 \%)$ had a specific/known cause. The most frequent related causes were hypoxic ischemic encephalopathy (HIE) (25.6\%) and neurocutaneous syndrome (17.9\%) with structural etiology (Figure 2).

According to the semiology of IS (Table 1), IS in flexion $(51.2 \%)$ and mixed (35.8\%) were the most frequent findings. IS were asymmetric in only three patients, being that two of them had agenesis of the corpus callosum and one porencefalic cyst secondary to ischemic CVD (cerebrovascular disease), although they also had symmetric IS. In four patients (10.2\%), IS were associated in particular with discognitive motor focal crises (upward gaze deviation, palpebral ptosis and disconnection). The symmetric characteristics of
Table 1. Clinical characteristics and findings in the electroencephalogram

\begin{tabular}{cc}
\hline Total (39) & \\
\hline Range of IS & $(15-350)$ \\
\hline Mode & 106 \\
\hline Semiology of IS & $\mathbf{N}(\%)$ \\
\hline Flexion & $20(51.2)$ \\
\hline Extension & $5(12.8)$ \\
\hline Mixed & $14(35.8)$ \\
\hline Associated with focal seizures & $4(10.2)$ \\
\hline Symmetric & $36(92.3)$ \\
\hline Asymmetric & $3(7.7)$ \\
\hline EEG & $\mathrm{N}(\%)$ \\
\hline Symmetric & $36(92.3)$ \\
\hline Asymmetric & $3(7.7)$ \\
\hline
\end{tabular}

EEG= Electroencephalogram; IS= Infantile spasms Source: Clinical history

hypsarrhythmia on EEG were verified in $92.3 \%$ of the cases. Subclinical IS (spindle - like) were observed in three patients.

Table 2 shows data on the clinical evolution of the studied children. Thirty of them (76.9\%) had abnormality in 
Table 2. Clinical evolution of the studied patients

\begin{tabular}{cc}
\hline Total (39) & N (\%) \\
\hline Moderate or severe delayed PD & $25(71.8)$ \\
\hline PD regression & $4(10.2)$ \\
\hline Lennox-Gastaut syndrome $^{\text {a }}$ & $14(35.9)$ \\
\hline Other epilepsies $^{*}$ & $17(43.6)$ \\
\hline Uncontrolled IS $^{*}$ & $21(53.8)$ \\
\hline Positive neurological examination & $30(76.9)$ \\
\hline Mild intellectual disability & $3(7.6)$ \\
\hline Death & $1(2.6)$
\end{tabular}

PD = Psychomotor development; IS= Infantile spasms

a-5 with Autistic Spectrum Disorder; ${ }^{*}-10$ with focal and seven with multifocal

Source: Clinical history

neurological PE from the beginning of IS. Four children (10.2\%) had a regression of the PD, being that five developed ASD. At the school age, nine patients presented severe ID, 14 moderate ID and three mild ID. In accordance with the evaluation of the psychiatry and psychology services of our Center, two patients presented average normal intelligence, normal PD and PE. Ten of these patients had focal characteristics and seven had multifocal spikes (based on results of EEG). The deceased patient had Proteus syndrome and the cause of death was unrelated to WS (bronchopneumonia and respiratory failure).

When observing the relationship between the different therapeutic options and EEG (Figure 3), it was verified the use of ACTH combined with VGB (as a first choice therapy) in 25 patients $(64.1 \%)$, and of VGB alone or combined with other drugs in $14(35.9 \%)$. The patients who used VGB alone or combined with other drugs were those who had inborn error of metabolism ( $n=1$ ) or other contraindications to the use of ACTH (including acute respiratory or diarrheal infections and infested skin lesions), as well as the children of cases that the parents did not give consent for the use of ACTH $(n=3)$.

Among all patients that used ACTH and VGB as a combined therapy, a control or decrease $\geq 50 \%$ of IS was verified in 18 of them $(72 \%)$ and, disappearance of IS and hypsarrhythmia in 14 (56\%). In four of these patients $(28.5 \%)$ only VGB was used (without the need for combination with other drugs), and in seven cases regardless of the use of combined treatment (due to the persistence of hypsarrhythmia and absence of total control of IS) were used other drugs in combination. Out of a total of 17 patients (43.5\%), seven (with use of ACTH and VGB) and 10 (with use of VGB and other drugs) had no response to the treatment, poor control of IS and persistent hypsarrhythmia despite the association of other drugs (polytherapy). When evaluating only the therapeutic response and EEG, it was noted that of the 25 patients who were treated from the beginning with combined treatment of ACTH and VGB, in $14(56 \%)$ there was disappearance of the hypsarrhythmia in the first 6 months. In two (14.2\%) of these 14 patients was also used VGB in combination with other drugs. In five patients the disappearance of the hypsarrhythmia was verified in the first 2 months (in four of them was used ACTH in combination with VGB). Statistical significance was observed between the groups $(p<0.05)$ regarding the used drugs and disappearance of hypsarrhythmia.

A better response was observed with the use of ACTH in combination with VGB in patients with known or symptomatic etiology ( $n=14)$ : five of them with neurocutaneous syndromes (except two with TSC (tuberous sclerosis complex)), two with genetic syndromes, two with CVD of prenatal origin, two with HIE and three with unknown etiology. The two patients who had the same response to the initial treatment with VGB alone presented hyperammonemia $(n=1)$ and $\operatorname{HIE~}(n=1)$.

Among all patients with initial combined treatment of ACTH and VGB, in 11 was also administered VA due to a low response to VGB or relapse. Moreover, benzodiazepines were used in four patients ( $n=2$ to CLB, $n=2$ to CLN) and TPM in three. Although polytherapy was used, seven of these patients did not respond well. Hypsarrhythmia persistence and evolution to LGS occurred. In this case, lamotrigine (LTG) was used in three patients. The most frequent adverse reaction due to the use of ACTH was transient hypertension $(n=25)$, while the less frequent were immunosuppression and infection $(n=2)$, and irritability ( $n=2)$. In addition, in 14 patients after completing 6 to 9 months of treatment with VGB monotherapy, this drug was replaced by VA (after clinical evaluation and EEG) and the treatment was adapted according to the evolution to electroclinical syndrome, except for the two patients with TSC. In the ophthalmological assessment there was no evidence of visual damage due to the use of VGB.

Of the 14 patients submitted to the initial treatment with VGB and others drugs, in 12 was also administered VA and other

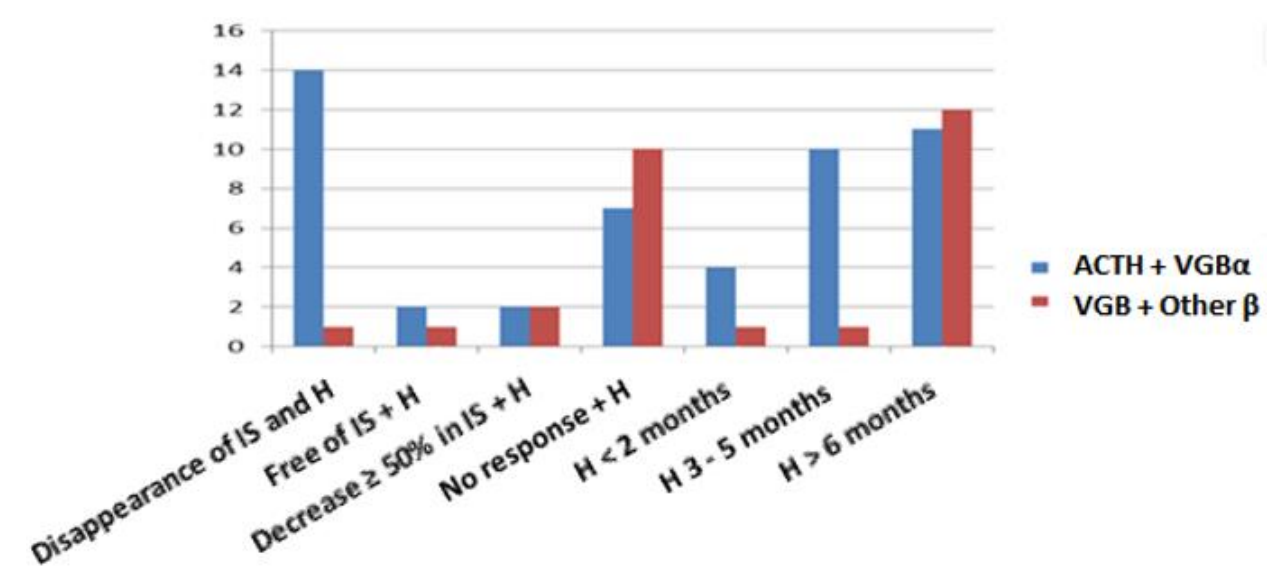

ACTH= Adrenocorticotropic Hormone; VGB= Vigabatrin; $\mathrm{H}=$ Hypsarrhythmia; IS= Infantile spasms

Response - therapeutic options: chi2 test $=9.92, p=0.019$; EEG: chi2 test $=6.63, p=0.034$

Figure 3. Relationship between the response to different therapeutic options and electroencephalogram

Source: Clinical history 
Table 3. Relationship between prognostic factors and clinical evolution

\begin{tabular}{|c|c|c|c|}
\hline Total (39) & Unfavorable evolution & Favorable evolution & $95 \% \mathrm{Cl}$ \\
\hline Male & $19(65.5 \%)$ & $3(30 \%)$ & $0.86(0.67-1.09)$ \\
\hline Pre-natal antecedents & $14(48.3 \%)$ & $4(40 \%)$ & $0.94(0.70-1.97)$ \\
\hline Perinatal antecedents & $15(38.5 \%)$ & $3(30 \%)$ & $0.89(0.68-1.18)$ \\
\hline Postnatal antecedents & $3(7.6 \%)$ & 0 & $0.74(0.62-0.89)$ \\
\hline $\begin{array}{l}\text { Previous delay of the PD and/or abnormality in the } \\
\text { neurological PE }\end{array}$ & $26(89.6 \%)$ & $3(30 \%)$ & $0.83(0.66-1.04)$ \\
\hline Previous epileptic seizures $^{*}$ & $21(72.4 \%)$ & $4(40 \%)$ & $0.89(0.69-1.14)$ \\
\hline Previous paroxysmal EEG & $21(72.4 \%)$ & $4(40 \%)$ & $0.89(0.69-1.14)$ \\
\hline Onset of IS $<4$ months & $15(51.7 \%)$ & 0 & $0.74(0.62-0.89)$ \\
\hline Symptomatic or known etiology & $26(89.6 \%)$ & $6(60 \%)$ & $0.84(0.54-1.33)$ \\
\hline Previous time lost $\geq 1$ month & $8(27.5 \%)$ & $1(10 \%)$ & $0.84(0.62-1.12)$ \\
\hline No response to monotherapy or combined therapy & $19(65.5 \%)$ & $2(20 \%)$ & $0.82(0.65-1.04)$ \\
\hline hypsarrhythmic EEG $>6$ months & $19(65.5 \%)$ & $1(10 \%)$ & $0.78(0.63-0.97)$ \\
\hline More than three factors & $20(68.9 \%)$ & $1(10 \%)$ & $0.78(0.63-1.97)$ \\
\hline $\begin{array}{l}\mathrm{Cl}=\text { Confidence intervals; } \mathrm{PD}=\text { Psychomotor developm } \\
\text { with neonatal crises } \\
\text { More than three factors: chi2 test }=3.97, \mathrm{p}=0.043 \\
\text { Source: } \text { Clinical history }\end{array}$ & $=$ = Physical examınatı & Electroencephalog & Infantile spasms; \\
\hline
\end{tabular}

drugs ( $n=3$ to TPM, $n=3$ to LVT and $n=2$ to CLB) due to a low response to VGB, with electro-clinical evolution to LGS observed in three patients that used LTG. Of the seven patients with unknown etiology who started the treatment with VB6 (in the absence of expected response), in four of them was used combined therapy, in three VGB and other drugs, and VB6 was also used in one patient with Down syndrome. VGB was stopped after 6 to 9 months of treatment according to clinical evolution and the EEG results. Adverse reactions to VGB were observed in two patients with weight gain and two with irritability, without need to withdraw the medication. In the ophthalmological assessment no visual damage due to the use of VGB was also verified for these patients. Of all the patients who used VA, only 1 had an adverse reaction to hyperammonemia, which was a reason for stopping the medication.

When describing the relationship between prognostic factors and clinical evolution (Table 3 ), we observed an unfavorable evolution in 29 patients $(74.4 \%)$, while 10 (25.6\%) had a favorable evolution. The most frequent risk factors in patients with unfavorable evolution were the symptomatic or known etiology, and delay of the PD and/or abnormality in the neurological PE $(n=26,89.6 \%)$. Among the patients with favorable evolution, the prognostic factors were present in less than $40 \%$ of the cases, with the exception of the known etiology $(n=6,60 \%)$.

\section{DISCUSSION}

IS are a classic component of the WS epileptic seizures. They usually are related to a devastating and unfavorable prognosis, which brings great uncertainties on the patient evolution to both the physicians and family members. Therefore, WS corresponds to the most serious and catastrophic epileptic encephalopathy after the neonatal period and in the first 2 years of life, because of the high probability of its evolution to intractable and drug-resistant epilepsy forms, with significant cognitive deficiencies, motor disabilities and severe neurodevelopmental impairment $(16,20,37,38)$.

It is also worth mentioning that IS are specific disorders associated with the age, which occur as a result of the neuronal excitability and cerebral immaturity due to various etiologies (genetic, metabolic, teratogenic, prenatal infectious and, perinatal and postnatal causes). A remarkable and variable number of individual predisposing factors are related to IS. In addition, regardless the moment of the beginning of the underlying insult (pre-, peri- and/or postnatal), IS begin at a different stage of development and/or as a "final common route" to all the described etiologies (typically between 3 and 7 months of life on average), with occurrence in a critical period of the common brain maturation (21). It is for this reason that any theory concerning this must respond to the different unknowns still existing:

How can a single disorder have different etiologies? Why do IS arise or occur only in childhood and independently of their causes? Why do their consequences have such an impact in all levels of neurodevelopment?

Little is known about the IS pathophysiology. Although the causes related to IS and their etiologies are usually variable, a common mechanism has been proposed concerning this subject. Current IS animal models focus on a specific cause of IS, such as the loss of interneurons and/or, inflammatory or immune responses (according to the findings in the ARX and TTX mouse models), stress and action of the corticotropinreleasing hormone ( $\mathrm{CRH})$. IS cause an increase in oxidative stress and promote release of $\mathrm{CRH}$ in the limbic, hypothalamic, amygdala and brainstem regions of rats with IS. Other animal models suggest the occurrence of a common mechanism for the loss of inhibition related to $\gamma$-aminobutyric acid (19-22). This explains the origin of IS, which occur due to a developmental abnormality or can be related to cortical or subcortical desynchronization (focal or diffuse), leading to an abnormal functional interaction with the brainstem (23).

Both the usual age of onset of IS and their slight predominance in males are data confirmed from different sources $(3,4,7,14,16)$. In our study we observed onset of IS between 4 and 8 months of age in the majority of the cases, with a slight predominance in males. Regarding the semiology of IS and EEG, there is a consensus that the symmetric axial contraction of muscles occurs in flexor muscles in about $40 \%$ of cases, or as a mixed contraction in 50\% (with symmetric hypsarrhythmic EEG) $(3,4,14,15)$. In our patients the flexor IS was predominant in comparison to the mixed IS. Although we also observed focal seizures associated to a smaller number of 
cases (4), such finding has also been evidenced by other researchers $(4,5,11)$.

With the advent of new neuroimaging technologies and, metabolic and genetic studies based on a correct PE and adequate clinical history, it has been possible to achieve the clarification of the etiology related to WS in most cases $(3,16)$. In connection with this, it has been demonstrated the occurrence of symptomatic or known origin in about $80 \%$ of patients affected with IS. According to the new classification proposed by $\operatorname{ILAE}(5,6)$, this is due to the genetic and structural/metabolic causes.

In our research, a similar percentage was observed. The etiology was classified as known in $82 \%$ of the cases and as unknown in $18 \%$ (despite the limitations existing in the health system of Cuba). The prenatal and perinatal causes were the most commonly related to the described etiology, including HIE (with its structural sequels), neurocutaneous syndromes, especially TSC, metabolic and genetic errors, and CVD of pre and perinatal origin. This has also been reported in several studies $(3-5,9,14,16,17,20)$.

In this sense, a greater importance should be attributed to NMR, which allows to define and visualize sequential structural alterations in HIE (such as periventricular leukomalacia and multicystic encephalomalacia) and CVD (such as poroencephalic cysts), as well as lesions described in different neurocutaneous syndromes, including TSC and Sturge-Weber Syndrome, such as was previously reported by Arce-Portillo et al (7) and Galicchio et al (17). It is noteworthy that, despite we have had only a 0.35 tesla NMR system, in this study we were able to identify other alterations like neuronal migration disorders (5.1\%) and agenesis of the corpus callosum (5.1\%). Therefore, at present, it would be relevant the use of a 1.5 tesla NMR system or higher to the elucidation of the number (percentage) of alterations that still remains unknown and, from that, to reveal new findings, as for example the occurrence of cortical dysplasias and neuronal migration disorders (18).

Another important point is that the overall prognosis of WS is conditioned by the etiology and epileptic activity, which is why the affected children require psycho-pedagogical support and rehabilitation during their development $(16,20,37,38)$. More than $70 \%$ of patients with WS present previous abnormalities in neurological PE and PD delay, others have regression in $\mathrm{PD}$ (posteriorly), and those with a previous $\mathrm{PD}$ abnormality after the onset of IS lose the skills they had acquired untimely $(16,20)$, with ID of up to $90 \%$ and comorbidity with ASD $(4,7,15,16,20)$. In our findings the rate of patients that had a similar behavior was $67 \%$, with five patients with ASD.

Furthermore, 55 to $60 \%$ of patients with this syndrome have frequent relapses, poor control of IS and subsequently develop other types of epilepsies, such as LGS and focal epilepsies with focal dyscognitive seizures or, motor, consciousness alterations (or others), with multifocal spikes on EEG $(7,15,16,31-33)$. In our study, $53.8 \%$ of the patients did not have a good control of IS and, $79 \%$ evolved to other epileptic syndromes ( $35.9 \%$ to LGS and $43.6 \%$ to other epilepsies) together with a higher proportion of symptomatic or known etiologies. In the patients with detected multifocal spikes, the focal dyscognitive seizures (motor or non-motor) were predominant, with evolution sometimes to bilateral seizures without evidence of generalized seizures during sleep and/or their predominance, since these children did not present typical clinical features of LGS (they were in a possible transition state, or not even that), with good response to VA and benzodiazepines.

It should be also noted that in our study there were two patients with average intellectual quotient that had unknown etiology and a good response to the treatment, without relapse and remission of IS and hypsarrhythmia in less than 2 months. Moreover, they presented normal PD after control of IS and neurological PE without alterations. At present, these patients are without treatment with antiepileptics and with absence of evolution to other syndromes. Therefore, according to the old etiological classification we could say that these would be two idiopathic cases.

Thus, an important question arises: Are the current therapies for IS capable of promoting significant improvements in patients with WS, or they simply treat a symptom and/or clinical sign? The most effective current therapies (ACTH and/or VGB) do not suppress suddenly the occurrence of IS, but rather gradually over the course of 2 to 4 weeks (20). It has been reported an average delay of 2 days after the initiation of ACTH therapy to the suppression of IS as well as the improvement of hypsarrhythmia in the posterior EEG, proposing that transcriptional and plastic changes are important to the therapeutic effects of ACTH, with disappearance of 80 to $88 \%$ of IS in patients treated with use of this drug. On the other hand, at least $40 \%$ of patients that respond to this treatment can have relapses, which suggests that epileptogenesis has not been inhibited $(21,23)$. The combination with VGB could prevent such relapses in patients that respond to this treatment, since there would be action of this drug by a different and inhibitory mechanism of $\gamma$ aminobutyric transferase (21-23). In this case, WS should be an example to demonstrate the electro-clinical correlation in the evaluation of the therapeutic response $(7,8,14,16,31,33)$.

The efficacy of ACTH (not necessarily at high doses) for the rapid and complete reduction of IS has been demonstrated in prospective controlled studies (23). In rat animal model has been observed that ACTH produces glucocorticoid release and, most of its effects are attributed to the activation of glucocorticoid receptors in the central nervous system by a "common excitatory pathway", similarly to what occurs with the various etiologies of IS and the activation of the "stress system". Steroids administered as therapy or secreted by the adrenal glands after the administration of ACTH decrease the release of $\mathrm{CRH}$ and have direct action on melanocortin receptors, which can to reduce and/or inhibit the neuronal excitability. Moreover, in some patients the suppression of IS and hypsarrhythmia improve the neurodevelopment in the long term, while in others the neurological deficit and/or abnormality of PD persist. This happens due to the pathogenic mechanisms underlying the etiology and other independents, which are not modified by the used medication (21-24).

According to several prospectives studies $(7,8,14,16,19,26,31,35)$ VGB should be the first-line medication for the treatment of IS, especially in patients with WS and TSC. With a dose/response effect between 100 to $200 \mathrm{mg} / \mathrm{kg} /$ day, it is possible with use of VGB to achieve the suppression of IS in up to $95 \%$ of patients with TSC and in $54 \%$ of those with other etiologies (7). A rapid response (between 3 and 5 days) and control of IS between 1 to 3 weeks of treatment may also be expected $(7,19,26)$.

On the other hand, ACTH therapy have been the first-line treatment for IS since 2005, given that was demonstrate that it 
exceed the effectiveness of VGB in 2 weeks of treatment (7). In other retrospective and prospective studies $(23-25,30,34)$ ACTH was also the first- line treatment for IS. As there is no difference in the response to the treatment using high or low doses of ACTH, it has been proposed the use of low doses to avoid adverse reactions more commonly related to the synthetic ACTH, at doses $0.0125 \mathrm{mg} / \mathrm{kg} /$ day $(4,7,25,28,30,31)$. There is still no single consensus on the duration of its use, although is acceptable the administration during 1 to 6 weeks, and in maximum within 12 weeks $(7,25,28,30,31)$. With use of this hormonal therapy is possible to suppress IS in $42 \%$ to $87 \%$ of cases, with an efficacy in the response to the treatment between 7 to 12 days after the beginning of the treatment (7).

There are prospective studies (but they are inconclusive), such as the International Collaborative Infantile Spasms Study (7), which propose the combined use of both drugs from the beginning of the treatment of IS, reducing thus the time of use of ACTH and possible adverse reactions, with established dose of VGB, and lower number of relapses $(29,36,39-41)$. In the present study, we used both ACTH and VGB as drugs of choice and in combination in $64.1 \%$ of the patients. $56 \%$ of them became free of IS and the hypsarrhythmia disappeared on EEG in the first six months, which coincides with the results from other researches $(36,39)$. In addition, TAH was observed as the more frequent adverse effect (without hemodynamic repercussion in the total of the treated patients) causing in two patients immunosuppression and respiratory infection. However, TAH was treatable and reversible in all affected patients, with absence of cardiovascular affectation demonstrated by ultrasound, and consequently no reason to suspend the use of the drugs. The combined therapy was used at low doses and not exceeding 6 weeks, as recommended by other authors, and the observed adverse effects were similar with other previous studies $(7,8,16,23,26,28,30)$. This therapy was more effective to the group with known/symptomatic or genetic/structural-metabolic etiology ( $\mathrm{n}=5$ with neurocutaneous syndrome and $n=2$ with TSC), in the treatment of CVD, and in $50 \%$ of the cases of unknown or probably symptomatic etiology.

Of the $35.9 \%$ patients that used VGB (alone) as initial treatment for the reasons previously cited, only two achieved disappearance of IS and hypsarrhythmia in the first six months. Therefore, in our study there was a better response with the use of the combined therapy. Of these patients, those with postinfectious etiology, HIE and unknown or probably symptomatic etiology had little response. Moreover, irritability and weight gain were observed as an adverse effect in $14 \%$ of these cases, and an adjustment of dose was done. The presence of irritability was difficult to determine if it was due to medication or WS, which corroborates the findings of ArcePortillo et al (7). During the neuro-ophthalmological follow-up no evidence of visual alterations attributed to medication was observed.

Regarding the total number of treated patients (with ACTH or VGB alone, or in combination), $58.9 \%$ achieved control or a reduction of IS to $50 \%$, with disappearance of hypsarrhythmia in $41 \%$ in the first 5 months of treatment. These findings are also sustained by the results of the study of Arce-Portillo et al (7), which demonstrated efficacy of both drugs, alone or in combination.

In patients who did not respond well to the combined therapy or alone with VGB, other drugs, such as VA and TPM, or LVT and benzodiazepines were used to a lesser extent, according to our therapeutic availability and following the recommendations of other authors $(3,5,8,16,27,31)$. VB6 was used as initial therapy in patients with unknown etiology and in cases of Down syndrome, as advocated by Campistol et al (16) and Caraballo et al (31). A greater emphasis was directed to the cases of unknown etiology, and no favorable response was observed. This led us to suggest that error of metabolism should not be a probable etiology in most of our patients. In addition, we did not use other alternative therapies recommended for non-responders to the treatment or to those patients with drug-resistant WS (as for example the use of ketogenic diet or achievement of injury and functional surgery) $(4,5,8,14,16,31)$, since such therapies have not been offered by our health system. It would be important the use of these alternative treatments in future researches or new health programs.

We found that although $74.4 \%$ of our patients have been treated with use of choice therapies, they had an unfavorable evolution, without much difference with the results found by Arce-Portillo et al (7). This is due in large part to the presence of factors of poor prognosis, such as patients with delay in the PD and/or abnormality of neurological PE prior to the onset of IS, epileptic seizures beginning in the neonatal period or a posteriori, previous paroxysmal EEG, and symptomatic or known etiology. A worse prognosis was observed among patients with HIE, postinfectious etiology and their structural sequels. Two of these patients presented neuronal migration disorders, one had kernicterus, three neurocutaneous syndrome ( $\mathrm{n}=2$ with TSC) and other three, an unknown etiology.

Another relevant aspect in this study was that when the age of onset of IS was less than 4 months and the hypsarrhythmia persisted on EEG after 6 months and/or there was a combination of more than three prognostic factors, it was observed statistical differences in IS alone or combined with other alterations, coinciding with the findings of Arce-Portillo et al (7).

Moreover, some authors noted to be relevant the time until the start of treatment when the diagnosis is made after the first month of the beginning of IS and the response to the drugs of choice is poor $(7,16,31)$. In this sense, it should be taken into account the repercussion that these factors could have to PD, cognitive and /or behavioral spheres, as well as concerning the evolution to other epileptic syndromes later, especially LGS with onset before the age of 2 years $(7,16,31,37,38)$. These characteristics also were observed in our patients.

By contrast, in patients with a favorable prognosis (25.6\%), there was a lower presence of these prognostic factors. None of the patients had an early onset of IS and in only one of them more than one month of treatment was lost. In another the hypsarrhythmia persisted after six months on EEG and two had poor response to monotherapy or combined therapy. Moreover, a combination of more than three prognostic factors was observed in only one patient, which also corroborates the findings of Arce-Portillo et al (7).

In short, we observed (regarding our 39 cases of infants with WS followed by 11 years) the occurrence of IS with a greater frequency between 4 and 8 months of age, with predominance in males and, symmetry on EEG concerning the semiology of IS in flexion or mixed and hypsarrhythmia in most cases. The symptomatic or known (structural/metabolic and genetic) etiologies were the more prevalent. HIE and neurocutaneous syndromes were the most common etiologies 
related to our cases of WS. When the therapeutic protocol existing in the hospital was used, more than half of the cases had a control of IS or their reduction greater than $50 \%$, with disappearance of the hypsarrhythmia in the first 5 months.

It was also observed a greater response to the combined therapy of ACTH and VGB, which demonstrated efficacy of both drugs as therapy of choice. The use of ACTH also showed efficacy in the suppression/reduction of IS in an early manner and improvement or disappearance of hypsarrhythmia on EEG. Furthermore, the adverse effects of ACTH were treatable, transient and reversible, since this drug was used in low doses and for period less than 6 weeks. Other drugs, such as VA, TPM, LVT and benzodiazepines were in our context remain alternative therapies to non responders to the initial therapies, while VB6 was administered in patients with unknown etiology and suspicion of metabolic error associated with deficiency of this vitamin.

The majority of our patients had an unfavorable prognosis regardless of the etiology. There was a worse prognosis in those who had previous to the onset of the delay in the PD and/or neurological abnormality to $\mathrm{PE}$, neonatal or a posteriori epileptic seizures, paroxysmal previous EEG, early onset of IS (before 4 months), persistence of hypsarrhythmia on EEG after 6 months, start of treatment after one month of onset of IS and poor therapeutic response.

This demonstrates that the repercussion of these factors of poor prognosis (alone or in combination) is determinant for an unfavorable evolution including severe forms with delayed PD, moderate or severe ID and association with ASD with evolution to other epileptic encephalopathies, such as LGS and epilepsies with multifocal spikes on EEG. Moreover, both the use of choice treatments and the control of IS do not ensure a favorable evolution, since the outcomes depend on the related etiology, epileptic activity, individual clinical characteristics and presence of factors of poor prognosis.

Finally, the conductance of multicentric studies may be needed to improve the knowledge about the variables as well as used therapies, in order to homogenize the therapeutic interventions and improve the follow-up for patients with WS.

\section{REFERENCES}

1. D’Alonzo R, Rigante D, Mencaroni E, Esposito S. West Syndrome: a review and guide for paediatricians. Clin Drug Investig 2018;38(2):113-24. https://doi.org/10.1007/s40261 -017-0595-z PMid:29086890

2. West WJ. On a peculiar form of infantile convulsions. Lancet 1841;35(911):724-5. https://doi.org/10.1016/S0140-6736 (00)40184-4

3. Pozo Alonso AJ, Pozo Lauzán D, Pozo Alonso D. Síndrome de West: etiología, fisiopatología, aspectos clínicos y pronósticos. Rev Cubana Pediatr 2002;74(2):151-61.

4. Taghdiri MM, Nemati $\mathrm{H}$. Infantile spasm: a review article. Iran J Child Neurol 2014;8(3):1-5.

5. Lux AL. Lastest American and European updates on infantile spasms. Curr Neurol Neurosci Rep 2013;13(3):334. https://doi.org/10.1007/s11910-012-0334-z PMid:23341252
6. Berg AT, Berkovic SF, Brodie MJ, et al. Revised terminology and concepts for organization of seizures and epilepsies: report of the ILAE Commission on Classification and Terminology, 2005-2009. Epilepsia 2010;51(4):676-85. https://doi.org/10.1111/j.1528-1167.2010.02522.x PMid:20196795

7. Arce-Portillo E, Rufo-Campos M, Muñoz-Cabello B, et al. Síndrome de West: etiología, opciones terapéuticas, evolución clínica y factores pronósticos. Rev Neurol 2011;52(2):81-9. https://doi.org/10.33588/rn.5202.2010392 PMid:21271547

8. Fejerman N. Síndrome de West: certezas, incógnitas y sorpresas. In: Ruggieri VL, Caraballo RH, Arroyo HA, editors. Temas de neuropediatría. Homenaje al Dr. Natalio Fejerman. Buenos Aires: Editorial Médica Panamericana; 2005:5-41.

9. Paciorkowski AR, Thio LL, Dobyns WB. Genetic and biologic classification of infantile spasms. Pediatr Neurol 2011;45(6):355-67. https://doi.org/10.1016/j.pediatrneurol. 2011.08.010 PMid:22114996 PMCid:PMC3397192

10. Proposal for revised classification of epilepsies and epileptic syndromes. Commission on Classification and Terminology of the International League Against Epilepsy. Epilepsia 1989;30(4):389-99. https://doi.org/10.1111/j.1528 -1157.1989.tb05316.x PMid:2502382

11. Fejerman N. Diagnósticos diferenciales del síndrome de West. Rev Neurol 2013;57(Supl 1):S125-8. https://doi.org/10.33588/rn.57S01.2013247

12. Caraballo RH, Ruggieri V, Gonzalez G, et al. Infantile spams without hypsarrhythmia: a study of 16 cases. Seizure 2011;20(3):197-202. https://doi.org/10.1016/j.seizure.2010. 11.018 PMid:21167750

13. Salar S, Moshé SL, Galanopoulou AS. Metabolic etiologies in West syndrome. Epilepsia Open 2018;3(2):134-66. https://doi.org/10.1002/epi4.12102 PMCid:PMC5983207

14. Dulac O, Lassonde M, Sarnat HB. Handbook of Clinical Neurology. Pediatric Neurology Part I. Elsevier 2013;111:861.

15. Fisher RS, van Emde Boas W, Blume W, et al. Epileptic seizures and epilepsy: definitions proposed by the International League Against Epilepsy (ILAE) and the International Bureau for Epilepsy (IBE). Epilepsia Epilepsia 2005;46(4):470-2. https://doi.org/10.1111/j.0013-9580.2005 .66104.x PMid:15816939

16. Campistol J, García-Cazorla A. Síndrome de West. Análisis, factores etiológicos y opciones terapéuticas. Rev Neurol 2003;37(4):345-52.

https://doi.org/10.33588/rn.3704.2003181

17. Galicchio S, Cersósimo R, Caraballo RH, Yépez-E II, Medina C, Fejerman N. Resonancia magnética cerebral en el estudio del síndrome de West. Rev Neurol 1999;28(7):685-7. https://doi.org/10.33588/rn.2807.98908 PMid:10363294

18. Lin DDM, Gallagher A. Advances in pediatric epilepsy neuroimaging. Journal of Pediatric Epilepsy 2013;2(01):1-2.

19. Carmant L. Vigabatrin therapy for infantile spasms: review of major trials in Europe, Canada, and the United States; and recommendations for dosing. Acta Neurol Scand Suppl 2011;124(192):36-47. https://doi.org/10.1111/j.1600-0404. 2011.01599.x PMid:22061179 
20. Wheless JW, Gibson PA, Rosbeck KL, et al. Infantile spasms (West syndrome): update and resources for pediatricians and providers to share with parents. BMC Pediatr 2012;12:108. https://doi.org/10.1186/1471-2431-12-108 PMid:22830456 PMCid:PMC3411499

21. Chudomelova L, Scantlebury MH, Raffo E, Coppola A, Betancourth D, Galanopoulou AS. Modeling new therapies for infantile spasms. Epilepsia 2010;51 Suppl 3:27-33. https://doi.org/10.1111/j.1528-1167.2010.02605.x PMid:20618396 PMCid:PMC2909007

22. Watemberg N. Infantile spasms: Treatment challenges. Curr Treat Options Neuro 2012;14(4):322-31. https://doi.org/10.1007/s11940-012-0181-x PMid:22581010

23. Brunson KL, Avishai-Eliner S, Baram TZ. ACTH treatment of infantile spasms: mechanisms of its effects in modulation of neuronal excitability. Int Rev Neurobiol 2002;49:185-97. https://doi.org/10.1016/S0074-7742(02)49013-7

24. Brunson KL, Eghbal-Ahmadi M, Baram TZ. How do the many etiologies of West syndrome lead to excitability and seizures? The corticotropin releasing hormone excess hypothesis. Brain Dev 2001;23(7):533-8. https://doi.org/10.1016/S0387-7604(01)00312-6

25. Wray CD, Benke TA. Effect of price increase of adrenocorticotropic hormone on treatment practices of infantile spasms. Pediatr Neurol 2010;43(3):163-6. https://doi.org/10.1016/j.pediatrneurol.2010.04.005 PMid:20691936 PMCid:PMC3197710

26. Fejerman N, Cersósimo R, Caraballo R, et al. Vigabatrin as a first-choice drug in the treatment of West syndrome. J Child Neurol 2000;15(3):161-5. https://doi.org/10.1177/ 088307380001500304 PMid:10757471

27. González JE, Martínez A, Avendaño M, et al. Guía práctica clínica. Tratamiento farmacológico del Síndrome de West. Rev Chil Epilepsia 2006;7(1):42-7.

28. Sakakihara Y. Treatment of West syndrome. Brain Dev 201;33(3):202-6. https://doi.org/10.1016/j.braindev.2010. 12.004 PMid:21196092

29. O'Callaghan FJ, Lux AL, Darke K, et al. The effect of lead time to treatment and of age of onset on developmental outcome at 4 years in infantile spasms: evidence from the United Kingdom Infantile Spasms Study. Epilepsia 2011, 52(7):1359-64. https://doi.org/10.1111/j.1528-1167.2011. 03127.x PMid:21668442
30. Nakata M, Kato T, Ide M, et al. Long-term weekly ACTH therapy for relapsed West syndrome in tuberous sclerosis complex: a case report. Brain Dev 2016;38(4):431-4. https://doi.org/10.1016/j.braindev.2015.10.004 PMid:26482603

31. Caraballo RH, Fejerman N. Tratamiento de las epilepsias: Buenos Aires: Editorial Médica Panamericana;2009:292.

32. Yacubian EMT, Kochen S. Crises epilépticas. São Paulo: Leitura Médica Ltda.;2014:96.

33. Caraballo RH, Cersósimo RO. Atlas de electroencefalografía en la epilepsia. Buenos Aires: Editorial Médica Panamericana;2010.

34. Zhu X, Chen O, Zhang D, et al. A prospective study on the treatment of infantile spasms with first-line topiramate followed by low-dose ACTH. Epilepsy Res 2011;93(2-3):14954. https://doi.org/10.1016/j.eplepsyres.2010.12.002 PMid:21205578

35. Gaily E. Vigabatrin monotherapy for infantile spasms. Expert Rev Neurother 2012;12(3):275-86. https://doi.org/10.1586/ern.12.3 PMid:22364326

36. Hancock EC, Osborne JP, Edwards SW. Treatment of infantile spasms. Cochrane Database Syst Rev 2013;5(6):CD001770. https://doi.org/10.1002/14651858. CD001770.pub3

37. Durá-Travé T, Yoldi-Petri ME, Hualde-Olascoaga J, EtayoEtayo V. Epilepsias y síndromes epilépticos durante el primer año de vida. Rev Neurol 2009;48(6):281-4. https://doi.org/10.33588/rn.4806.2008446 PMid:19291649

38. Barbarrosa EP. Características de la epilepsia antes del primer año en el Hospital Pediátrico de Centro Habana durante el período 2004-2009. Rev Cubana Neurol Neurocir 2012;2(2):121-8.

39. Riikonen R. Recent advances in the pharmacotherapy of infantile spasms. CNS Drugs. 2014; 28(4):279-90. https://doi.org/10.1007/s40263-014-0139-5 PMid:24504827

40. Farnosova ME, Zharikova TF, Gamirova RG, Sivkova SN. Timing of treatment initiation in West's syndrome. Int J Risk Saf Med 2015;27(1):S55-S6. https://doi.org/10.3233/ JRS-150688 PMid:26639711

41. Kelley SA, Knupp KG. Infantile Spasms-Have We Made Progress? Curr Neurol Neurosci Rep 2018;18(5):27. https://doi.org/10.1007/s11910-018-0832-8 PMid:29671077 